\title{
Metric discrimination and distribution of the species of Crocidura occuring in Tunisia
}

\author{
Maurizio SARÀ and Laura ZANCA
}

Sarà M. and Zanca L. 1992. Metric discrimination and distribution of the species of Crocidura occuring in Tunisia. Acta theriol. 37: 103 - 116.

A recent paper on the occurrence of the genus Crocidura in Tunisia reports a single specimen identified as $C$. suaveolens. Therefore a third species, besides $C$. russula and $C$. whitakeri would occur in the country. However, the presence of $C$. suaveolens in North-Africa is controversial and was recently ruled out from the other Maghrebi countries (Algieria and Morocco). During the period 1989 - 90, 71 specimens of shrews were collected from owls pellets or trapped at Tunisian 12 sites. This material was measured and studied both by classic morphometric and multivariate methods (Fuzzy test, Principal Coordinate Analysis and Generalized Procrustes Analysis), considering also reference samples ( $C$. suaveolens from Italy and Balearic Islands, C. russula and C. whitakeri from Morocco). Four of the 12 trapped shrews were karyotyped and resulted to have a $C$. russula karyotype, some differences in biometry as well as in Number of Fundamental arms (NFa) separate the Moroccan population from the Tunisian one and raise some interesting questions on the taxonomy of $C$. russula. Multivariate analysis also allowed us to exclude the presence of $C$. suaveolens from the Tunisian material studied and suggested also the misclassification of the proposed specimen that should be reconsidered as $C$. whitakeri. A preliminar distribution map of the two species living in Tunisia was drawn, also based on the scanty data from the literature.

Institute of Zoology, University of Palermo, Via Archirafi, 18, 90123 Palermo, Italy

Key words: Crocidura, multivariate analysis, distribution, Tunisia

\section{Introduction}

This paper represents a contribution to the study of the controversial taxonomic relationships between $C$. suaveolens and C. whitakeri in North-Africa. Some authors (for a review see: Vesmanis 1988, Kowalski and Rzebik-Kowalska 1991) considered $C$. whitakeri as a subspecies of $C$. suaveolens, but this interpretation is no longer accepted because other studies support the opinion that $C$. whitakeri is a separate species. The presence of $C$. whitakeri and the absence of $C$. suaveolens in the northern part of Morocco (Hutterer 1987) and in Algeria (Rzebik-Kowalska 1988) has in fact been confirmed. In Tunisia the former species and C. russula had already been recorded (cfr. Heim de Balsac 1968), but recently Vesmanis (1988) has assigned a single specimen to C. suaveolens. The purpose of this study 
was to check the presence of C. suaveolens in our Tunisian collection and draw a preliminar distribution of the genus in the country.

\section{Materials and methods}

During three field trips all over Tunisia $(1989$ - 1990) a fairly abundant number of Barn owl (Tyto $a l b a$ ) and Great owl (Bubo bubo) pellets were collected. Pellets contained remains of Crocidura skulls, as listed in Table 1. A trapping survey (June 1990) proved successful only in a northern site where 12 shrews were caught. Some of the living shrews were air-mailed to Prof. P. Vogel (Lausanne) for biochemical and karyological analyses, the other specimens were stored in the theriological collection of the Zoological Museum of Palermo University (MZPA).

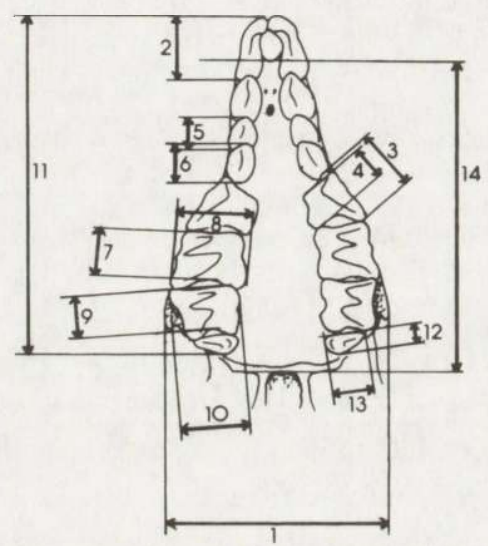

Fig. 1. The 14 skull measurements, according to Rzebik-Kowalska (1988). Measurements 12 and 13 on the third molar $\left(\mathrm{M}^{3}\right)$ were excluded from the multivariate analysis because of the common loss of this tooth in pellet remains.

Table 1. Number of individuals and skull remains collected from Barn owl (Tyto alba) and Eagle owl (Bubo bubo) pellets and by trapping $(*)$, at 12 Tunisian sites (**). The Lac Mellègue individual was found dead in 1979 and stored in the MZPA collection. $C$. indet. corresponds to very broken material.

\begin{tabular}{lrrrrrrr}
\hline \multirow{2}{*}{ Locality } & \multicolumn{9}{c}{ N indiv. } & Calvaria & \multicolumn{2}{c}{ Mandibles } & \multicolumn{2}{l}{} \\
& & & right & left & C. $r$. & C. $w$. & C. indet. \\
\hline Aïn Draham $\left(^{*}\right)$ & 12 & 12 & 2 & 12 & 12 & 0 & 0 \\
Bordj Fedjadj & 5 & 4 & 4 & 5 & 0 & 5 & 0 \\
Lac Mellègue $\left(^{* *}\right)$ & 1 & 1 & 1 & 1 & 1 & 0 & 0 \\
Roman aqueduct & 8 & 7 & 7 & 8 & 8 & 0 & 0 \\
Dj. Zaghouan & 9 & 6 & 8 & 5 & 5 & 3 & 1 \\
Tabarka & 5 & 4 & 2 & 5 & 4 & 0 & 1 \\
Kasserine & 12 & 5 & 7 & 11 & 5 & 5 & 2 \\
Bulla Regia & 11 & 9 & 11 & 9 & 9 & 2 & 0 \\
Kef el Garia & 1 & 0 & 0 & 1 & 0 & 1 & 0 \\
Tamerza & 1 & 1 & 0 & 1 & 0 & 1 & 0 \\
Sbetïla & 0 & 0 & 0 & 0 & 0 & 0 & 0 \\
Maktar & 6 & 6 & 6 & 6 & 0 & 6 & 0 \\
Total & 71 & 45 & 48 & 54 & 44 & 23 & 4 \\
\hline
\end{tabular}


The material has been measured according to Rzebik-Kowalska (1988) by means of a Citoval binocular with micrometric lenses, and aged according to Vesmanis and Vesmanis (1979); skull measurements taken are shown in Fig. 1. Eighteen graded non-metric characters of the mandible and lower dentition (Butler and Greenwood 1979, Butler et al. 1989) were used to investigate the relationships between the two species in a set of six species (C. suaveolens, C. whitakeri, $C$. russula, C. leucodon, C. sicula and C. canariensis).

Four of the trapped specimens were karyotyped in Lausanne, and their skulls were employed to check the identity of the remaining tunisian pellet specimens following Rzebik-Kowalska's method, based on tooth ratios (width/length) and on the variable ranges overlap. The set of 12 skull variables, which is reported in Appendix 1, was then processed by multivariate analysis. For this analysis the classification and ordination routines of the SYN-TAX IV statistical package (Podani 1990a) were employed.

A ncn-hierarchical method of classification, the fuzzy c-means clustering, was first chosen for grouping 35 specimens ( 15 C. russula from Tunisia, 5 C. whitakeri from Tunisia, 1 C. whitakeri from Morocco, 14 C. suaveolens from Italy as outgroup); whereas the traditional clustering methods are often reported to produce artificial clusters, fuzzy clustering is less prone to produce such artefacts. The method calculates membership weights for every object to express, for a given coefficient of fuzziness, its affinity to each cluster. Its underlying strategy is the minimization of within-cluster sum of squares by an iterative algorithm. The coefficient of overall separation of clusters ranges from 0 to $\mathrm{m}$ (number of objects); the closer it is to $\mathrm{m}$ the "harder" the proportion is. Partition efficiency is measured by Dunn's partion entropy (Podani 1990a, b).

The second step in multivariate analysis was to check the metric ordination of the specimens in order to slear up the groups resulting from fuzzy clustering. The sample processed was thus increased from 35 to 62 specimens including 15 C. russula from Morocco, 1 from Algeria and 11 C. suaveolens balearice from Minorca (Balearic islands). The analysis run was the principal coordinates analysis (PCA), d the log-transformated variables, with six different distance coefficients; the consensus configurtion among the different ordinations was then calculated by generalized procrustes analysis (Prana).

As regards multivariate analysis, it is important to note that the small sample processed, can only result in a first definition of the biometric relationships among the taxa involved, therefore the canonica variates analysis, whose discriminating power depends on the sample size, is not reported.

Vesnanis (1988) gave measurements and drawings of a specimen, coming from the surroundings of Gebess, identified as $C$. suaveolens. The set of variables recorded by this Author is slightly different from the one used here (four variables were not recorded), but permitted to derive the tooth ratios accordin to Rzebik-Kowalska's method. By using the Gower's coefficient for missing data this specimen was also introduced in the multivariate matrix to be processed.

\section{Results}

\section{Biometric analysis}

The four specimens showed a $C$. russula karyotype of $2 \mathrm{n}=42, \mathrm{NFa}=68$ i.e. differert in the number of fundamental arms from the Moroccan and Swiss $C$. russula $(2 \mathrm{n}=42, \mathrm{NFa}=60)$ (Vogel et al. in press). The metric measurements taken in these animals and the Rzebik-Kowalska's (1988) taxonomic method, permitied us to recognize two different Tunisian taxa that would correspond to $C$. whitakri and $C$. russula respectively. In Table 2 we report the tooth ratios comparng the Tunisian skulls with the other taxa involved in the study. The tooth ratios ary among different populations but generally the first $\left(\mathrm{M}^{1}\right)$ and second 
Table 2. Range ( $\min -\max$ ) of tooth ratios in different populations of shrews. The width/lengit ratio (b/a) of the first and second molars are usefull for discrimination. The Vesmanis' specimen lies in the C. whitakeri range. $\left({ }^{*}\right)$ values taken from Rzebik-Kowalska (1988).

\begin{tabular}{llcccc}
\hline Species & Country & Ratio A1/A2P & Ratio b/a $\mathrm{M}^{1}$ & Ratio b/a M & Ratio b/a M \\
\hline C. russula & Tunisia & $1.95-2.86$ & $1.60-1.95$ & $1.73-2.06$ & $1.93-2.60$ \\
C. russula & Morocco & $1.95-2.47$ & $1.63-1.91$ & $1.70-2.10$ & $1.92-2.70$ \\
C. russula & Algeria (*) & $1.69-2.19$ & $1.84-2.20$ & $1.78-2.11$ & $2.18-2.23$ \\
C. suaveolens & Italy & $1.89-2.85$ & $1.64-1.95$ & $1.70-1.95$ & $1.79-2.17$ \\
C. suaveolens & Minorca & $2.19-2.92$ & $1.67-1.82$ & $1.60-2.00$ & $1.67-2.60$ \\
C. suaveolens & Poland (*) & $1.84-2.33$ & $1.51-1.87$ & $1.71-1.93$ & - \\
C. whitakeri & Tunisia & $2.33-2.89$ & $1.89-2.24$ & $2.11-2.40$ & $2.11-2.78$ \\
C. whitakeri & Morocco & $1.95-2.64$ & $2.03-2.26$ & $2.12-2.25$ & $2.00-2.49$ \\
C. whitakeri & Algeria (*) & $2.18-3.04$ & $2.20-3.00$ & $2.09-2.54$ & $2.09-2.10$ \\
Vesmanis' specimen & & 2.35 & 2.11 & 2.17 & 2.36 \\
\hline
\end{tabular}
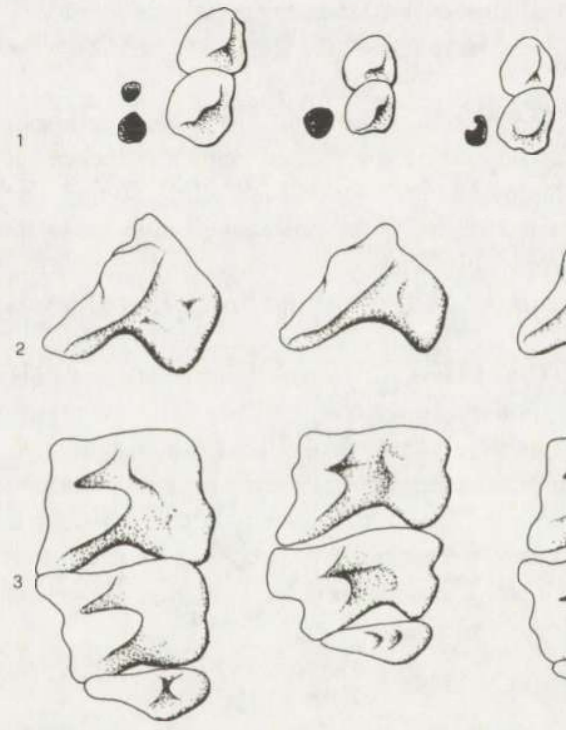
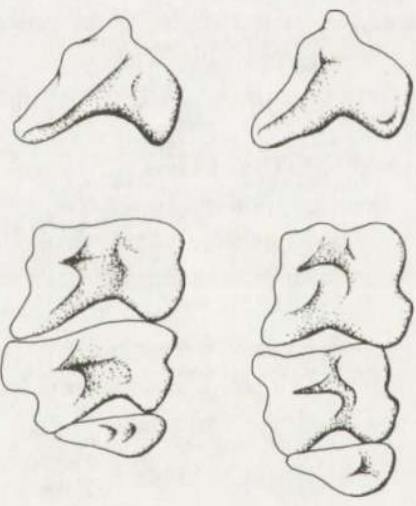

Fig. 2. Different shapes of teeth (in occlusal view) useful for Tunisian Crocidura skull determination $(\mathrm{R}=$ russula, $\mathrm{W}=$ whitakeri); these characters are constant over the Maghrebi populations. $C$. suaveolens $(\mathrm{S})$ com: from penninsular Italy. $1=\mathrm{A}^{2}$ and $\mathrm{A}^{3}$ Antenolars, next to the $\mathrm{A}^{3}$ hole-roots; $2=\mathrm{P}^{4}$ Prenolar; $3=\mathrm{M}^{1}-\mathrm{M}^{3}$ Molars.

$\left(\mathrm{M}^{2}\right)$ molar ratios are useful in discriminating among $C$. whitakeri and the other two species. During this preliminar work the taxonomic attribution of specmen no. 10 coming from Lac Mellègue (Appendix 1), was rather problematic. In this specimen some skull charcters, according to the current taxonomic keys, did not match to C. russula (e.g. palatal and zygomatic lengths), but the mandible vas a "typical" C. russula. The skull morphology distinguished also fairly well $C$. suaveolens from $C$. whitakeri. Some characters, reported in Fig. 2 such as the number and shape of $\mathrm{A}^{3}$ roots, the teeth shape and size $\left(\mathrm{A}^{2}<\mathrm{A}^{3}\right.$ in $C$. suaveslens 
Table 3. Membership weights for each specimen obtained by fuzzy classification.

\begin{tabular}{|c|c|c|c|}
\hline Ind. & Cluster 1 & Cluster 2 & Cluster 3 \\
\hline 1 & 1.0000 & 0.0000 & 0.0000 \\
\hline 2 & 1.0000 & 0.0000 & 0.0000 \\
\hline 3 & 0.9999 & 0.0000 & 0.0001 \\
\hline 4 & 1.0000 & 0.0000 & 0.0000 \\
\hline 5 & 1.0000 & 0.0000 & 0.0000 \\
\hline 6 & 1.0000 & 0.0000 & 0.0000 \\
\hline 7 & 0.9997 & 0.0000 & 0.0003 \\
\hline 8 & 1.0000 & 0.0000 & 0.0000 \\
\hline 9 & 1.0000 & 0.0000 & 0.0000 \\
\hline 10 & 0.0040 & 0.0017 & 0.9943 \\
\hline 11 & 0.9998 & 0.0000 & 0.0002 \\
\hline 12 & 1.0000 & 0.0000 & 0.0000 \\
\hline 13 & 1.0000 & 0.0000 & 0.0000 \\
\hline 14 & 1.0000 & 0.0000 & 0.0000 \\
\hline 15 & 1.0000 & 0.0000 & 0.0000 \\
\hline 16 & 0.0000 & 0.0001 & 0.9999 \\
\hline 17 & 0.0000 & 0.0002 & 0.9998 \\
\hline 18 & 0.0000 & 0.0001 & 0.9999 \\
\hline 19 & 0.0001 & 0.2265 & 0.7734 \\
\hline 20 & 0.0000 & 0.0000 & 1.0000 \\
\hline 21 & 0.0037 & 0.0015 & 0.9948 \\
\hline 22 & 0.0000 & 0.0004 & 0.9996 \\
\hline 23 & 0.0002 & 0.1877 & 0.8121 \\
\hline 24 & 0.0000 & 0.1039 & 0.8960 \\
\hline 25 & 0.0070 & 0.0058 & 0.9873 \\
\hline 26 & 0.0001 & 0.0002 & 0.9997 \\
\hline 27 & 0.0007 & 0.0014 & 0.9978 \\
\hline 28 & 0.0000 & 0.0007 & 0.9993 \\
\hline 29 & 0.0000 & 0.0019 & 0.9981 \\
\hline 30 & 0.0000 & 0.9990 & 0.0010 \\
\hline 31 & 0.0000 & 1.0000 & 0.0000 \\
\hline 32 & 0.0001 & 0.9588 & 0.0411 \\
\hline 33 & 0.0000 & 0.9999 & 0.0001 \\
\hline 34 & 0.0000 & 0.9847 & 0.0152 \\
\hline 35 & 0.0000 & 0.9876 & 0.0124 \\
\hline
\end{tabular}

and $\mathrm{A}^{2}>\mathrm{A}^{3}$ in C. whitakeri) are in fact very useful to separate the taxa considered. Analysis of the non-graded characters of the mandible and of the lower dentition provided similar results. The position of the mental foramen, the length and shape of the lingual cingula of $\mathrm{M}^{1}-\mathrm{M}^{3}$ and the talonid basin of $\mathrm{M}^{3}$ gave different scores, according Butler et al. (1989), and in a cladogram of shared derived characters $C$. suaveolens and $C$. whitakeri appear as separated lineages among the six taxa considered (Sarà and Zanca, in prep.). 
Table 4. Other results of fuzzy c-means clustering. It is interesting to note the low separation coefficients among the three taxa.

Partition defined by the highest weight for each individual:

Cluster 1: ind. from 1 to 9 and from 11 to $15=$ C. russula

Cluster 2: ind. from 30 to 35

$=$ C. whitakeri

Cluster 3: ind. from 16 to $29+$ ind. 10

=C. suaveolens + outlier C. russula

Coefficient of overall separation $=33.96$

Partition entropy

$$
=0.054
$$

Pairwise separation coefficients for clusters

\begin{tabular}{lccc}
\hline & C. $r$. & C. $w$. & C.s. \\
\hline C. $r$. & 0.00 & 0.63 & 0.36 \\
C. $w$. & - & - & 0.12 \\
\hline
\end{tabular}

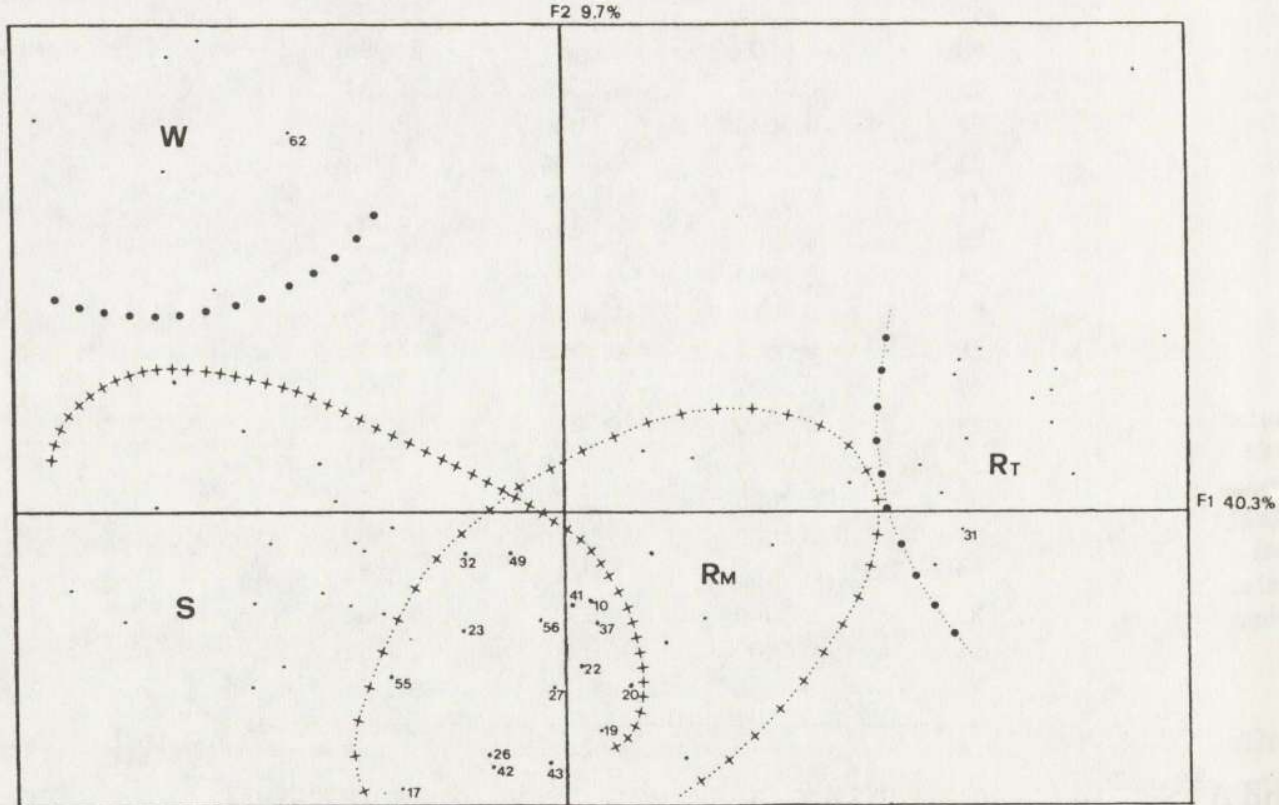

Fig. 3. The generalized procrustes chart shows the final configuration among six different coefficients of PCA. The scattergram displays the ordination, on the F1-F2 axes, showing the separation between the taxa. $\mathrm{W}=C$. whitakeri, $\mathrm{RT}=C$. russula from Tunisia, $\mathrm{RM}=C$. russula from Morocco, $\mathrm{S}=C$. suaveolens from Italy and Minorca.

Individuals coded as in Appendix $1.10=C$. russula from Tunisia, 17, 19, 20, 22, 23, 26, $27=C$. russula from Morocco, $31=$ C. russula from Algeria, 32, 37, 41, 42, $43=$ C. suaveolens from Italy, 49 , $55,56=$ C. suaveolens from Minorca, C. whitakeri from Tunisia, $62=C$. whitakeri from Morocco. 


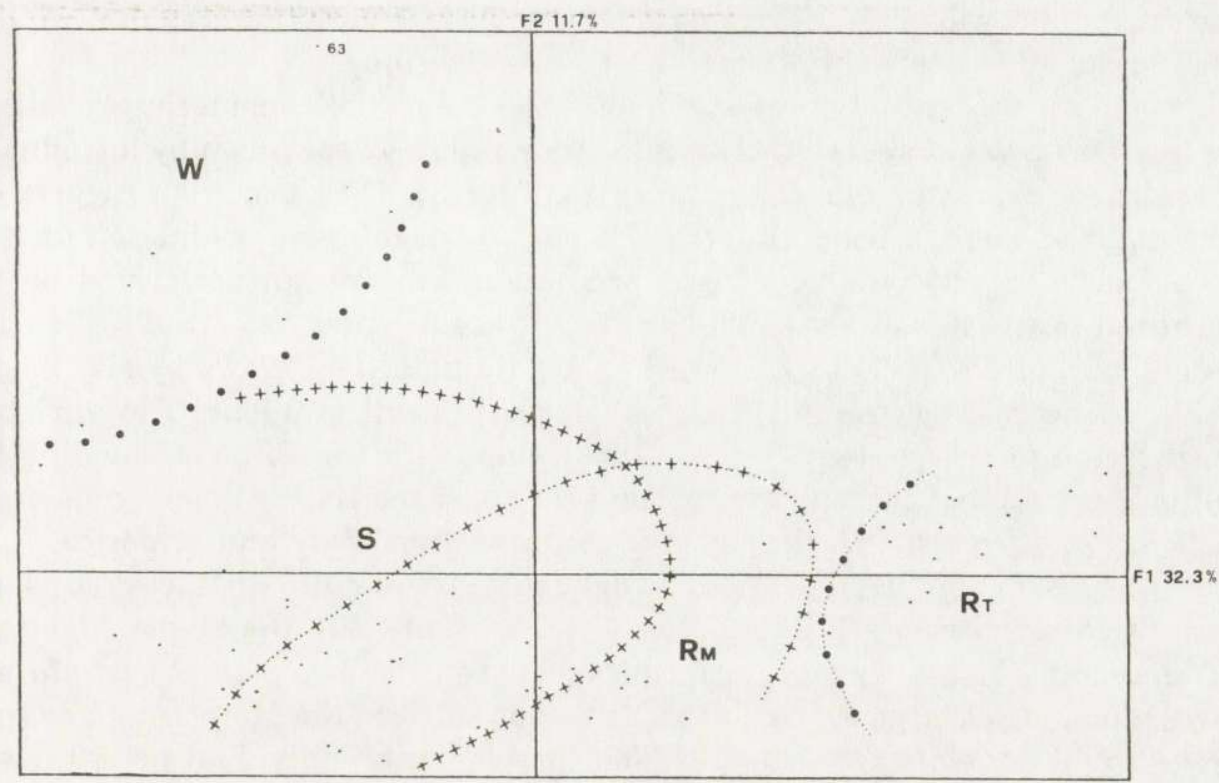

Fig. 4. Principal coordinate analysis, by Gower's coefficient for missing data, show how the Vesmanis' specimen (63) lies in the $C$. whitakeri biometric area. Symbols as in Fig. 3.

As regards the Vesmanis' specimen the $\mathrm{M}^{1}-\mathrm{M}^{3}$ tooth ratios proved to lie in the $C$. whitakeri range and not in the $C$. suaveolens one. Furthermore Fig. 3 on page 80 of Vestmanis' paper shows that $\mathrm{A}^{2}>\mathrm{A}^{3}$, as it normally occurs in $C$. whitakeri.

\section{Multivariate biometric analysis}

Tables 3 and 4 report the fuzzy clustering results; for the given coefficient of fuzziness (1.2) the whole sample of 35 individuals shows high afinity $(>0.75)$ to one of the three clusters. The resulting partition raised three different clusters and considering the taxonomic identification previously done, it was possible to recognize cluster 1 as $C$. russula, cluster 2 as $C$. suaveolens and cluster 3 as $C$. whitakeri.

The coefficient of overall separation of clusters has a high value (34 vs 35$)$ and the Dunn's partition entropy value, or resulting system fuzziness, is thus low; these results indicate that the hard separation of the 35 shrews into three taxa is meaningful.

The pairwise separation coefficients for clusters show the biometrical proximity of $C$. suaveolens and C. whitakeri, whereas there is a higher separation between these species and $C$. russula.

Shrew no. 10, according to fuzzy clustering could be placed in cluster 2, therefore another $C$. suaveolens specimen could be present in a Tunisian site, other than the one proposed by Vesmanis (1988). On the other hand the fuzzy clustering 
of the mandible, which is not reported here, gave a different results, in that mandible no. 10 clustered well with the $C$. russula group.

To clear up the allocation of specimen no. 10 and the considering results of fuzzy clustering we increased the sample processed to 62 specimen by introducing some related taxa available: C. russula from Morocco (15), and from Algeria (1), C. suaveolens balearica from Minorca (11), and by running an oridnation method (PCA). Fig. 3 displays the consensus ordination scattergram performed by the generalized procrustes analysis of six different coefficients.

Some variables (e.g. 1, 11, 12, see Fig. 1), ordinate the shrews by size on the F1 axis, along a largest (right) to smaller (left) skull gradient. The variables related to the teeth size (e.g. $3,5,7,9$ ) correlate with the F2 axis, dividing the small individuals into two sub-groups, and set apart the six specimens considered as $C$. whitakeri from the $C$. suaveolens specimens from Italy and Minorica.

The $C$. russula sample appears to be divided into two quite distinct groups, the Moroccan shrews being separated from the Tunisian (plus the single Algerian). The Moroccan C. russula lie intermediate between the Tunisian C. russula and the $C$. suaveolens. A large overlap area is present between the Moroccan C. russula and the whole $C$. suaveolens sample. The "problematic" individual no. 10 lies in this area and should be now better considered as a "Moroccan-like" C. russula. In Fig. 4 we display the PCA chart, once the Vesmanis' specimen (no. 63) has been introduced. This skull clumps together to the six C. whitakeri specimens.

\section{Preliminary distribution and ecology data of Crocidura in Tunisia}

Fig. 5 shows the sites where shrew remains were collected by us and the locations known from the literature (Heim de Balsac 1968, Vesmanis 1972, 1988).

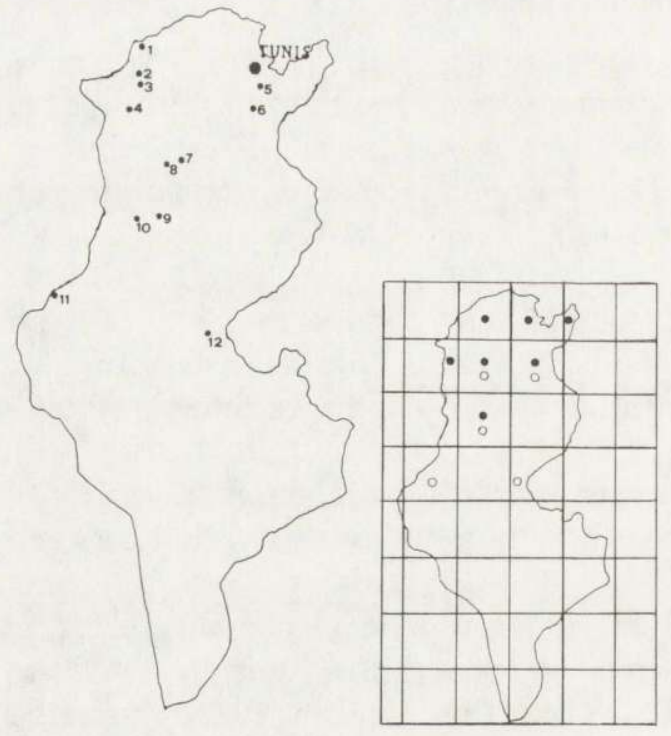

Fig. 5. The left map displays the sites of shrews collection: 1 = Tabarka, 2 = Aīn Draham, 3 = Bulla Regia, 4 = Lac Mellègue, $5=$ Roman aqueduct, $6=\mathrm{Dj}$. Zaghouan, $7=$ Kef el Garia, 8 = Maktar, 9 = Sbetīla, 10 = Kasserine, 11 = Tamerza, 12 = Bordj Fedjadj. While on the right there is the resulting distribution, including literature data. Black dots $=C$. russula, white dots $=C$. whitakeri . 
In Tunisia $C$. russula seems to have a northeastern distribution in wooded (Kroumiria, Medjerda) and agricultural landscapes where the yearly rainfall ranges from 200 to $400 \mathrm{~mm}$ and where the climate is mild, while C. whitakeri was collected more frequently in the semi-desertic areas with extensive grazing and alfa grass prairie.

The "C. suaveolens" specimen described by Vesmanis (1988) was collected in a typical arid habitat near Gabès. It represents the most southern specimen of shrew known for Tunisia. Very little information is, in fact, available for the southern area below the Chott el Djerid and Chott et Fedjadj line.

A large overlapping area probably exists along the border between the mediterranean and the semi-desertic areas (Kasserine-Dj. Zaghouan line) or where these landscapes are fragmented and intermingled, as in the central region. In these sites both species occur in pellets but this occurrence does not necessarily mean simpatry. It can depend also on Strigoformes, which in some cases (e.g. Kasserine) prey over heterogenous habitats such as irrigated plains, oases and the arid slopes of Djebel Chambi mountain, where the two species of shrews can occur separately. Finally of the 12 C. russula shrews trapped in the Ain Draham area; one came from an evergreen oak-wooded trap station, two from a dense maquis and nine from a ungrazed grassfield station.

\section{Discussion}

The purpose of the study was to investigate the relationships between $C$. whitakeri and C. suaveolens, as well as the presence of the latter species in the Tunisian material available, and finally verify the Vesmanis' attribution of the single specimen from Gabés to $C$. suaveolens. Our findings were a little bit more complicated owing to the presence of an outlier Tunisian specimen.

As regards the first aim, it is possible to conclude that $C$. whitakeri results to be biometrically quite distinct from $C$. suaveolens, but the final word about its specific status must still wait for karyological and biochemical confirmation, since the karyotype of $C$. whitakeri is not known yet. Further analysis of a larger sample of single populations will define better the ranges of biometrical characters and their population centroids and will thus permit to calculate robust discriminant equations between the two taxa.

As regards the Vesmanis' specimen, both the analyses feasible (tooth ratios and ordination with coefficient for missing data) with no direct handling of the material led us to argue for a misclassification and for a reattribution to C. whitakeri. C. suaveolens is not present, so far, among the 71 Tunisian shrews collected and studied by us, and it is unlikely to occur in this country.

More interestingly, the morphometric division resulting between the Tunisian and the Moroccan C. russula should be consistent with the difference already found in the C. russula $\mathrm{NFa}$ and permits to argue that $C$. russula is probably not a plain homogenous species, but rather a group of two (or more?) sibling species 
(Vogel et al., in press). Further karyotyping in the Lac Mellegué area (and elsewhere in Tunisia) should confirm this opinion, clearing up the genetic difference in the Tunisian C. russula population.

The biometric overlap among $C$. suaveolens populations and the Moroccan $C$. russula could be explained by characters displacement that would occur in these allotropic taxa.

Acknowledgements: We would like to thank J. Podani for his help in running the SYN-TAX IV package and for the useful comments on the manuscript; G. Sorci and R. Santolini are acknowledged for their help in collecting the material in Tunisia.

\section{References}

Butler P. M. and Greenwood M. 1979. Soricidae (Mammalia) from the Early Pleistocene of Olduvai Gorge, Tanzania. Zool. J. Linn. Soc. 67: $329-379$.

Butler P. M., Thorpe F. L. S. and Greenwood M. 1989. Interspecific relations of African Crocidurinae shrews (Mammalia: Soricidae) based on a multivariate analysis of mandibular data. Zool. J. Linn. Soc. $96: 373-412$.

Heim de Balsac H. 1968. Les Soricidae dans le milieux désertique saharienne. Bonn. zool. Beitr. 19: $181-188$.

Hutterer R. 1987. The species of Crocidura (Soricidae) in Morocco. Mammalia 50: 521 - 534.

Podani J. 1990a. SYN-TAX IV, user's manual. ICEM: 1 - 145.

Podani J. 1990b. Comparison of Fuzzy classifications. Coenoses 5: $17-21$.

Rzebik-Kowalska B. 1988. Studies on the genus Crocidura (Insectivora, Mammalia) in Algeria. Acta zool. Cracov. 31: 176 - 192 .

Kowalski K. and Rzebik-Kowalska B. 1991. Mammals of Algeria. Ossolineum, Wrocław: 1-370.

Vesmanis I. E. 1972. Einige Kleinsäuger vom Galita-Archipel, Tunesien. Senckberg. Biol. 53: 189 195.

Vesmanis I. E. 1988. Ein Nachweis der Gartenspitzmaus C. suaveolens (Pallas, 1811) in Tunesien. Abh. Mus. Tierkd. 14: $76-82$.

Vesmanis I. E. and Vesmanis A. 1979. Ein Vorschlag zur einheitlichen Altersabstufung bei Wimperspitzmäusen (Mammalia: Insectivora, Crocidura). Bonn zool. Beitr. 30: 7 - 13.

Vogel P., Maddalena T. and Sarà M. (in press). The taxonomic status of Crocidura cossyrensis Contoli, 1989 and its relationships to African and European Crocidura russula (Mammalia, Insectivora). In: Proceedings of the Sixth Int. Coll. on the Ecology and Taxonomy of Small African Mammals. Israel J. Zool.

Received 20 January 1992, accepted 26 May 1992. 


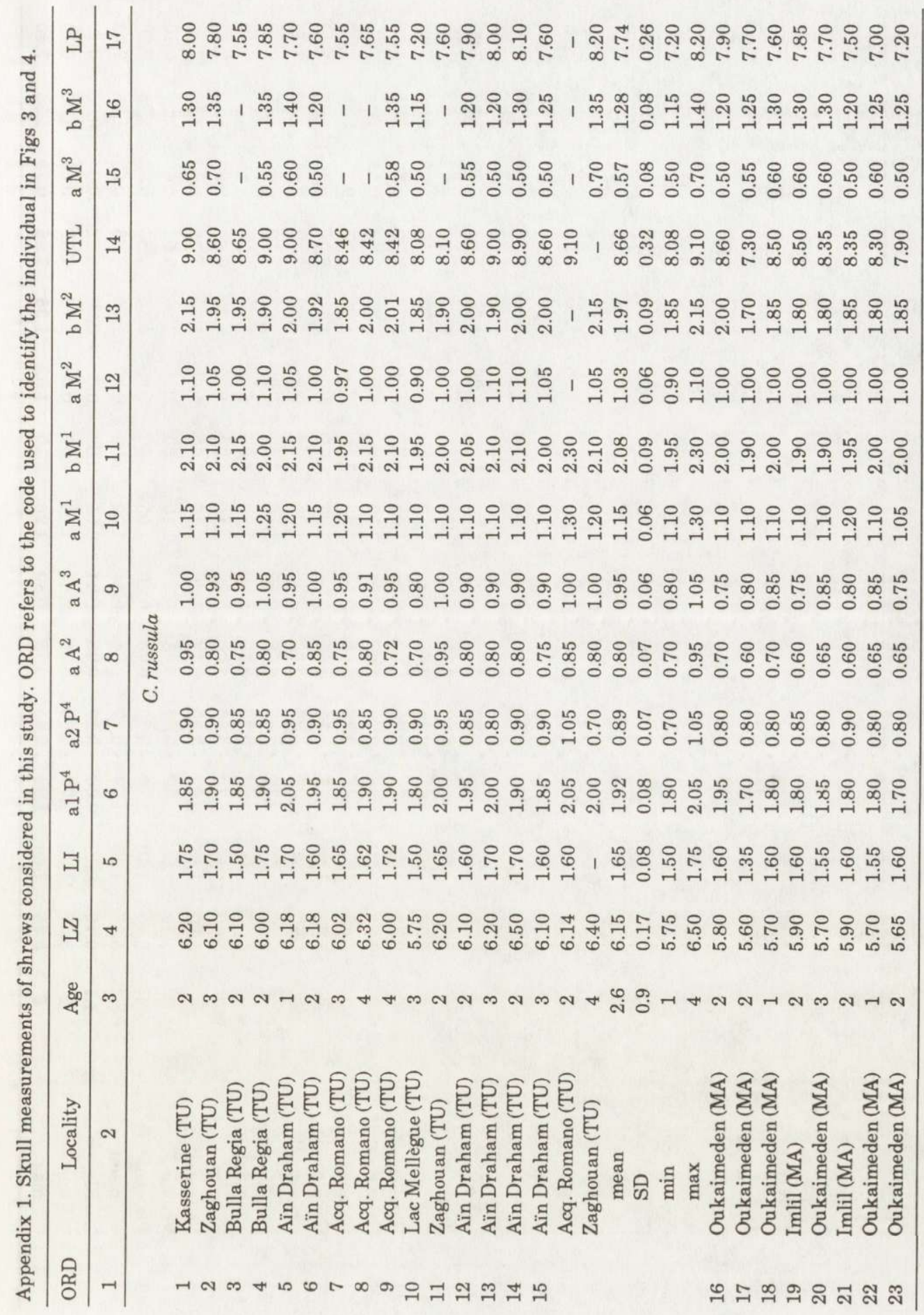




\begin{tabular}{|c|c|c|}
\hline ᄃ & 운 \& & 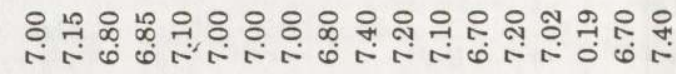 \\
\hline$\stackrel{-1}{-1}$ & 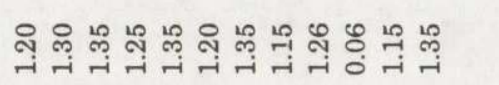 & 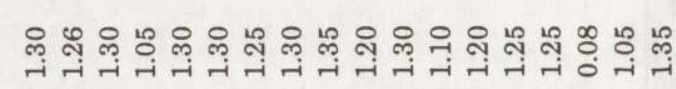 \\
\hline 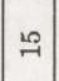 & 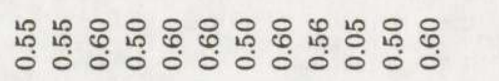 & $\begin{array}{lll}\sharp & 0 \\
0 & 0\end{array} 0$ \\
\hline 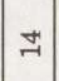 & 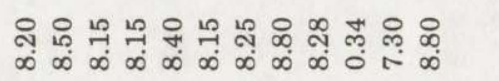 & \& \& \& \\
\hline$\dddot{\infty}$ & 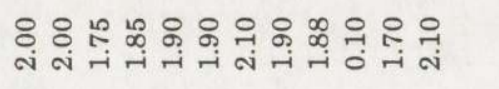 & 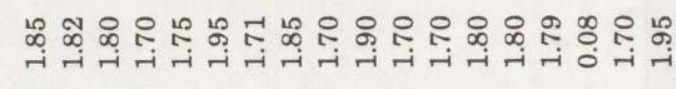 \\
\hline ㄱ & 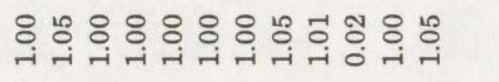 & 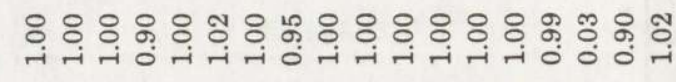 \\
\hline$\Rightarrow$ & 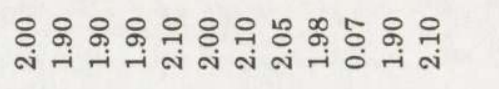 & 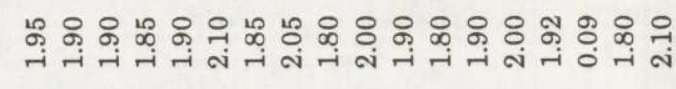 \\
\hline$ㅇ$ & 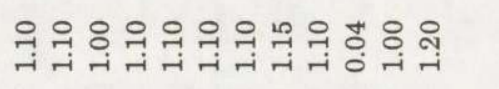 & 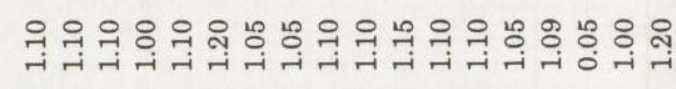 \\
\hline o) & 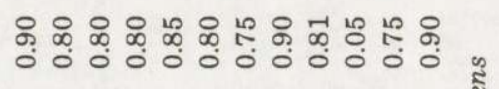 & 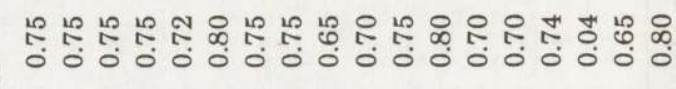 \\
\hline$\infty$ & 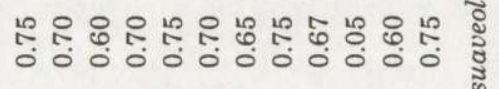 & 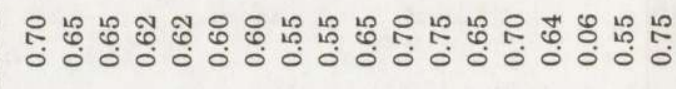 \\
\hline- & 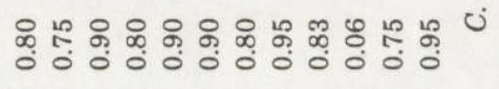 & 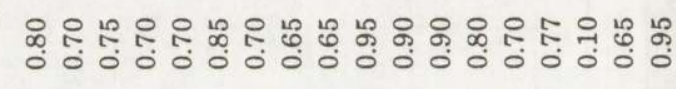 \\
\hline$\bullet$ & 点 & 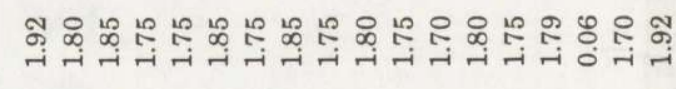 \\
\hline is & 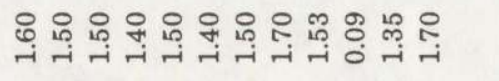 & 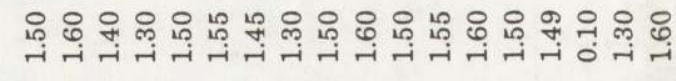 \\
\hline+ & 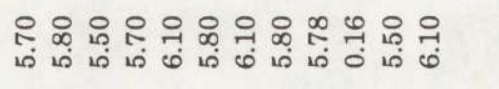 & 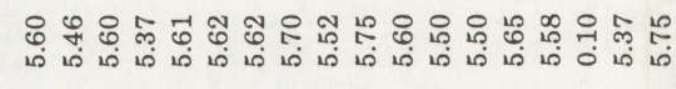 \\
\hline$\infty$ & Nล N & $\forall \forall \infty$ ถ \\
\hline ค & 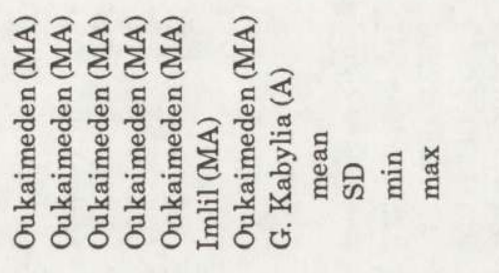 & 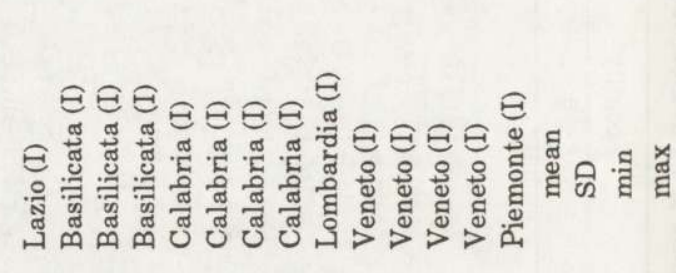 \\
\hline-1 & 茫 & : 8 \\
\hline
\end{tabular}




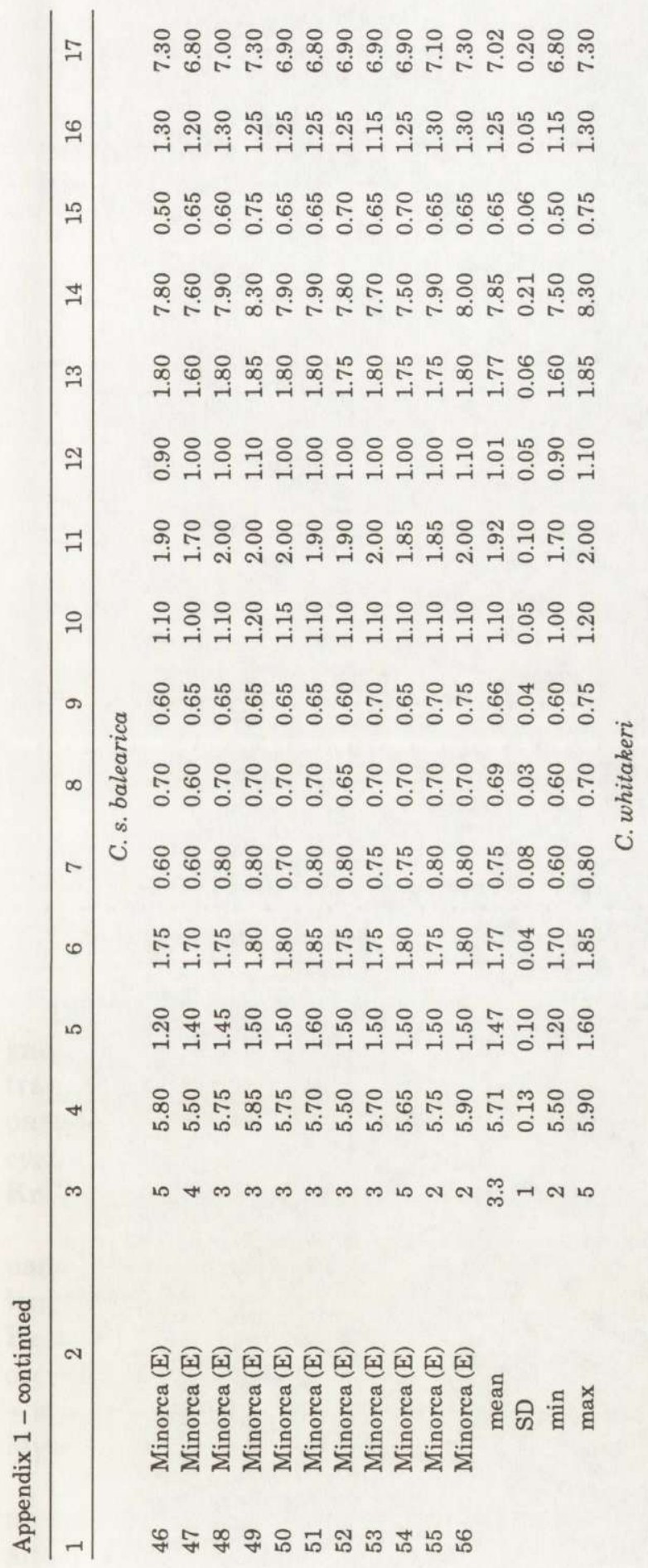

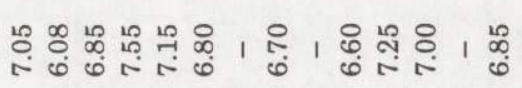

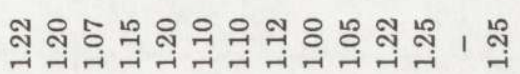

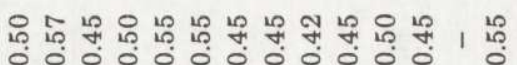
워

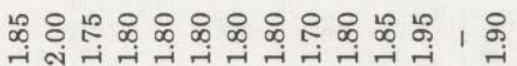

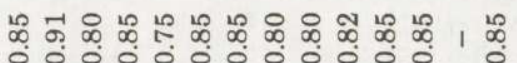

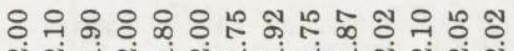

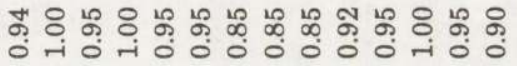

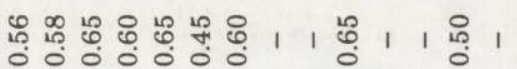

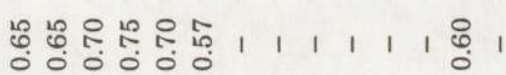

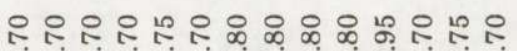
0000000000000

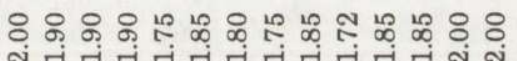
(1)

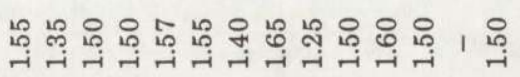

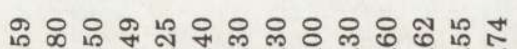

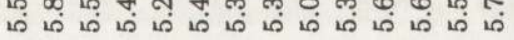

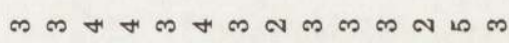

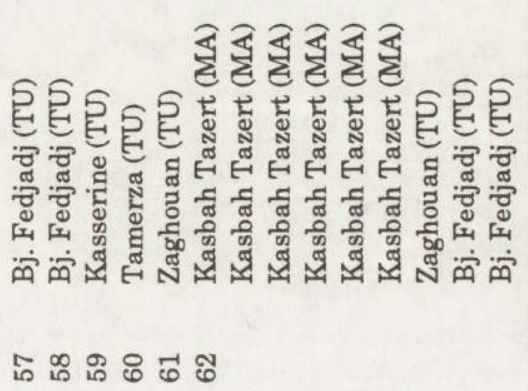


